Research Paper

\title{
A prognostic index model to individually predict clinical outcomes for colorectal cancer with synchronous bone metastasis
}

\author{
Xu Guan ${ }^{*}$, Chen-xi Ma1 ${ }^{*}$, Ji-chuan Quan ${ }^{1}$, Zhi-xun Zhao', Hai-peng Chen ${ }^{1}$, Peng Sun ${ }^{2}$, Song Wang ${ }^{2}$, Zhao \\ $\mathrm{Lu}^{1}$, Xiao-long Ma${ }^{1}$, Zheng Liu ${ }^{1}$, Zheng Jiang${ }^{1}$, Xi-shan Wang $^{1}{ }^{凶}$ \\ 1. Department of Colorectal Surgery, National Cancer Center/National Clinical Research Center for Cancer/Cancer Hospital, Chinese Academy of Medical \\ Sciences and Peking Union Medical College, Bejing, China \\ 2. Department of Colorectal Surgery, the Second Affiliated Hospital of Harbin Medical University, Harbin, China \\ *Xu Guan and Chen-xi Ma contributed equally to this work \\ $\triangle$ Corresponding author: Xi-shan Wang, Department of Colorectal Surgery, National Cancer Center/National Clinical Research Center for Cancer/Cancer \\ Hospital, Chinese Academy of Medical Sciences and Peking Union Medical College, Beijing, 100021, China. Tel: +86-13552367779; E-mail: \\ wxshan_1208@126.com. Zheng Jiang, Department of Colorectal Surgery, National Cancer Center/National Clinical Research Center for Cancer/Cancer \\ Hospital, Chinese Academy of Medical Sciences and Peking Union Medical College, Beijing, 100021, China. Tel: +86-18810817803; E-mail: 812818858@qq.com
}

() The author(s). This is an open access article distributed under the terms of the Creative Commons Attribution License (https://creativecommons.org/licenses/by/4.0/). See http:/ /ivyspring.com/terms for full terms and conditions.

Received: 2019.10.05; Accepted: 2020.02.20; Published: 2020.05.11

\begin{abstract}
Background: The prognosis of synchronous bone metastasis (BM) in colorectal cancer (CRC) is poor and rarely concerned. A clinical tool to evaluate the prognosis and clinical outcomes for BM would be attractive in current clinical practice.

Methods: A total of 342 CRC patients with synchronous BM were identified from Surveillance, Epidemiology, and End Results (SEER) database. The cancer specific survival (CSS) was estimated with the Kaplan-Meier method. Prognostic factors were identified from multivariate Cox model, and the final clinical nomogram was developed to predict the CSS. The concordance index (C-index) was used to assess the discriminative ability. Calibration curves were provided to internally validate the performance of the nomogram.

Results: The nomogram finally consisted of 6 prognostic factors including age, tumor grade, AJCC N stage, carcinoembryonic antigen (CEA) levels, primary tumor resection and chemotherapy, which translated the effects of prognostic factors into certain scores to predict the 1-, 2- and 3-year CSS for the synchronous BM in CRC patients. The nomogram presented a good accuracy for predicting the CSS with the $\mathrm{C}$-index of 0.742 . The calibration of the nomogram predictions was also accurate.

Conclusions: This nomogram was accurate enough to predict the CSS of CRC patients with synchronous BM using readily available clinicopathologic factors and could provide individualized clinical decisions for both physicians and patients.
\end{abstract}

Key words: colorectal cancer; bone metastasis; nomogram; prognostic factors; cancer specific survival; the Surveillance, Epidemiology, and End Results database

\section{Introduction}

Colorectal cancer (CRC) ranks the second most common cancer cause of death worldwide, leading to more than 881 thousand deaths in $2018^{(1)}$. Even bone metastasis $(\mathrm{BM})$ is relatively rare in $\mathrm{CRC}(2,3)$, the incidence of $\mathrm{BM}$ has gradually increased in recent years. BM is usually diagnosed at the advanced stages with a poor prognosis of 5-year survival rate with less than $5 \%(4)$, and the skeletal-related events (SREs) such as bone pain, pathological fracture, possible radiotherapy, spinal cord compression and fatal hypercalcemia could further affect the survival of patients(5). Therefore, the survival for each CRC 
patient with $\mathrm{BM}$ varies widely and there is no available evaluating system to provide prognosis prediction for this group of patients(6-8).

Previous clinical reports regarding the treatment of CRC patients with BM mainly concentrated in studies with small sample size and limited follow-up duration $(7,9,10)$, which may lead to big bias for clinical practice. Furthermore, current treatment plan making for systemic and local control of CRC patients with $\mathrm{BM}$ were mostly originated from clinical experience because of the relative rarity of BM in CRC patients. Hence, the current therapy for BM clearly lacks strong evidence and rigorous guidance.

Nomogram is a reliable and alternative tool to quantify risk by incorporating and illustrating crucial factors for prognosis and it has been proved to make more precise survival prediction compared with traditional TNM staging systems(11-13). In addition, nomogram could assist clinicians with making individual clinical decision making. Thus, the aim of our study was firstly to present the clinicopathological characteristics of CRC patients with BM from the nationwide population-based database, and then to develop a nomogram by utilizing readily available clinicopathologic factors, which could predict the prognosis and then potentially guide treatment decisions for CRC with synchronous BM.

\section{Materials and Methods}

\section{Data resources}

The patients with CRC diagnosed with synchronous BM were extracted from the Surveillance, Epidemiology, and End Results (SEER) database between January 2010 and December 2014. The SEER is an openly accessed database, which includes the information with regard to cancer incidence, survival outcome and treatment strategy from 17 population-based cancer registries and represents approximately 28 percent of the US population. Data in the SEER database do not require informed patient consent, because they were anonymized and de-identified prior to release. This study was approved by the Ethics Committee of National Cancer Center/National Clinical Research Center for Cancer/Cancer Hospital, Chinese Academy of Medical Sciences and Peking Union Medical College institutional review board.

\section{Study population}

All CRC patients included in this study were definitively diagnosed by pathological examination, and $\mathrm{BM}$ were diagnosed using imaging examination and/or pathological examination. The cancer-specific survival (CSS) was defined as the time from the diagnosis until cancer-associated death and the end of follow up. Several clinical and tumor related variables were collected to analyze the prognostic impact on survival, including age, gender, primary tumor location, tumor grade, tumor size, pathological type, CEA levels, AJCC $\mathrm{T}$ stage, AJCC $\mathrm{N}$ stage, extra-osseous metastasis (involving bone, brain, liver and lung metastasis), primary tumor resection, radiotherapy and chemotherapy in SEER database.

\section{Statistical analysis}

The CSS was assessed with Kaplan-Meier method, with the log-rank tests used to compare subgroups. In order to reduce the impact of sample size, potential prognostic factors with $\mathrm{P}<0.20$ in univariate Kaplan-Meier analyses ${ }^{(14)}$ were finally entered into multivariate analysis via the Cox regression model. The nomogram was developed based on these prognostic factors $(\mathrm{P}<0.05)$ from the final (after forward selection) Cox model to predict the CSS of CRC patients with synchronous BM, and discriminative ability was appraised by concordance index (C-index). Calibration curves, which plot the average Kaplan-Meier estimate against the corresponding nomogram for 1-, 2-, or 3-year predicted CSS were provided to internally evaluate the performance of the nomogram based on the Cox model. In evaluating calibration, we stratified the patients into 3 equally sized subgroups and bootstrap-corrected CSS rates were calculated by averaging the Kaplan-Meier estimates based on 1000 bootstrap samples. All statistical analyses were performed with SPSS version 25.0 for Mac and R version 3.6.0. It is considered as statistically significant when $\mathrm{P}<0.05$.

\section{Results}

\section{Patient characteristics}

After excluding 1,235 cases from SEER database who were not eligible, finally a total of 342 stage IV CRC patients with synchronous BM were conducted on our study (Figure 1), whose clinical and tumor characteristics were shown in Table 1. Patients with age $\geq 60(57.0 \%)$, colon cancer $(66.4 \%)$, low tumor grade $(63.1 \%)$, tumor size $\geq 5 \mathrm{~cm} \quad(58.5 \%)$, adenocarcinoma $(84.8 \%)$, CEA positive $(81.3 \%)$, high AJCC T stage $(85.4 \%)$ or lymph node metastasis positive $(77.2 \%)$ had higher proportion. Extra-osseous metastasis was present in the majority of cases $(78.4 \%)$, while the primary tumor was resected in $67.3 \%$ of cases. $116(33.9 \%)$ cases received radiotherapy while $226(66.1 \%)$ cases received chemotherapy in all patients. 


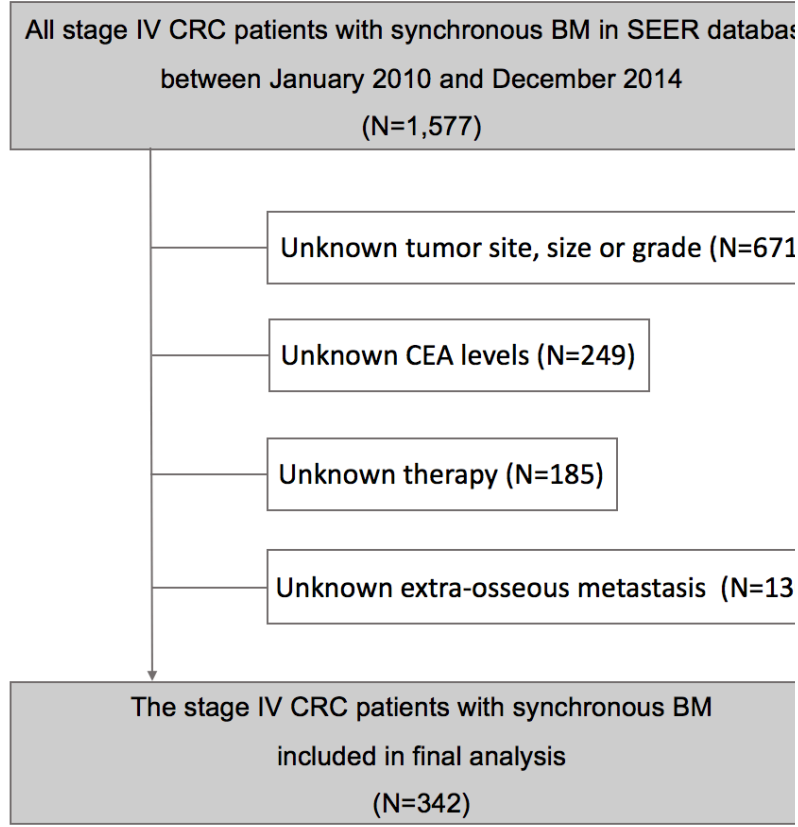

Figure 1 Analytical cohort and exclusion criteria of patients with synchronous bone metastasis from colorectal cancer.

\section{CSS analysis}

There was a total of $257(75.1 \%)$ patients died due to progressive CRC at the time of analysis. The median CSS in CRC patients with synchronous BM was 9 months, and the 1-, 2- and 3-year CSS of patients were $42.0 \%, 21.4 \%$ and $11.2 \%$. Each survival curve of potential prognostic variable with $\mathrm{P}<0.20$ in univariate Kaplan-Meier analyses were represented in Figure 2. These factors included age $(\mathrm{P}=0.001$; median CSS $=15$ and 6 months for age $<60$ and $\geq 60$, respectively), primary tumor site $(\mathrm{P}=0.066$; median CSS=7, 11 and 10 months for ascending colon, descending colon and rectum, respectively), tumor grade $(\mathrm{P}=0.000$; median $\mathrm{CSS}=13$ and 6 months for grade I, II and grade III, IV, respectively), pathological type $(\mathrm{P}=0.008$; median $\mathrm{CSS}=10,10,6$ and 3 months for adenocarcinoma, mucinous adenocarcinoma, signet-cell carcinoma and others, respectively), CEA levels $(\mathrm{P}=0.153$; median $\mathrm{CSS}=13$ and 9 months for CEA negative and positive, respectively), $\mathrm{N}$ stage $(\mathrm{P}=0.159$; median CSS $=14$ and 9 months for $\mathrm{N} 0$ and $\mathrm{N} 1, \mathrm{~N} 2$, respectively), primary tumor resection $(\mathrm{P}=0.004$; median CSS $=10$ and 7 months for with and without resection, respectively) and radiotherapy $(\mathrm{P}=0.150$; median $\mathrm{CSS}=10$ and 9 months for with and without radiotherapy, respectively). Especially, CSS was remarkable longer for patients who received chemotherapy compared to those who did not $(\mathrm{P}=0.000$; median CSS $=14$ and 3 months, respectively). The 1-, 2- and 3-year CSS of patients treated with chemotherapy were $54.0 \%, 27.8 \%$ and $14.6 \%$, comparing to $18.2 \%, 8.7 \%$ and $4.6 \%$ of 1 -, 2 - and 3-year CSS without chemotherapy, which have striking differences in survival.

Table 1. Clinical and tumor characteristics in colorectal cancer patients with synchronous bone metastasis.

\begin{tabular}{|c|c|c|c|}
\hline Characteristics & $\begin{array}{l}\text { Patient with BM } \\
(\mathrm{N}=342)\end{array}$ & $\%$ & $\begin{array}{l}\text { Univariable } \\
\text { Analysis P }\end{array}$ \\
\hline Age, years & & & .001 \\
\hline$<60$ & 147 & 43.0 & \\
\hline$\geq 60$ & 195 & 57.0 & \\
\hline Gender & & & .802 \\
\hline Male & 202 & 59.1 & \\
\hline Female & 140 & 40.9 & \\
\hline Primary tumor site & & & .066 \\
\hline Ascending colon & 146 & 42.7 & \\
\hline Descending colon & 81 & 23.7 & \\
\hline Rectum & 115 & 33.6 & \\
\hline Tumor grade & & & .000 \\
\hline Grade I, II & 216 & 63.1 & \\
\hline Grade III, IV & 126 & 36.9 & \\
\hline Primary tumor size, $\mathrm{cm}$ & & & .203 \\
\hline$<3$ & 36 & 10.5 & \\
\hline $3 \leq<5$ & 106 & 31.0 & \\
\hline$\geq 5$ & 200 & 58.5 & \\
\hline Pathological type & & & .008 \\
\hline Adenocarcinoma & 290 & 84.8 & \\
\hline $\begin{array}{l}\text { Mucinous } \\
\text { adenocarcinoma }\end{array}$ & 25 & 7.3 & \\
\hline $\begin{array}{l}\text { Signet-ring cell } \\
\text { carcinoma }\end{array}$ & 13 & 3.8 & \\
\hline Other & 14 & 4.1 & \\
\hline CEA & & & .153 \\
\hline- & 64 & 18.7 & \\
\hline+ & 278 & 81.3 & \\
\hline AJCC T stage & & & .289 \\
\hline $\mathrm{T} 1, \mathrm{~T} 2$ & 50 & 14.6 & \\
\hline $\mathrm{T} 3, \mathrm{~T} 4$ & 292 & 85.4 & \\
\hline AJCC N stage & & & .159 \\
\hline No & 78 & 22.8 & \\
\hline $\mathrm{N} 1, \mathrm{~N} 2$ & 264 & 77.2 & \\
\hline $\begin{array}{l}\text { Extra-osseous } \\
\text { metastasis }\end{array}$ & & & .392 \\
\hline No & 74 & 21.6 & \\
\hline Yes & 268 & 78.4 & \\
\hline $\begin{array}{l}\text { Primary tumor } \\
\text { resection }\end{array}$ & & & .004 \\
\hline No & 112 & 32.7 & \\
\hline Yes & 230 & 67.3 & \\
\hline Radiotherapy & & & .150 \\
\hline No & 226 & 66.1 & \\
\hline Yes & 116 & 33.9 & \\
\hline Chemotherapy & & & .000 \\
\hline No & 116 & 33.9 & \\
\hline Yes & 226 & 66.1 & \\
\hline
\end{tabular}

BM: bone metastasis; N: number; CEA: carcinoembryonic antigen 
The final multivariable Cox model analysis of CSS was presented in Table 2. The significant prognostic variables including age $(\mathrm{P}=0.011)$, tumor grade $(\mathrm{P}=0.000)$, primary tumor resection $(\mathrm{P}=0.000)$ and chemotherapy $(\mathrm{P}=0.000)$ was identified in final Cox analysis. Moreover, the model also included CEA levels $(\mathrm{P}=0.005)$ and AJCC $\mathrm{N}$ stage $(\mathrm{P}=0.001)$ as the independent factors for CSS in CRC with synchronous $\mathrm{BM}$, although neither were they significant in univariate Kaplan-Meier analyses $(\mathrm{P}=0.153$ and $\mathrm{P}=0.159$ respectively).

A nomogram developed by using the above six prognostic factors from the final multivariate Cox model was represented in Figure 3. The nomogram aimed to translate the effects of prognostic factors into certain scores and a weighted total score was calculated from these factors to predict the 1-, 2- and 3-year CSS for synchronous BM in CRC patients. Then we internally validated the nomogram which used the same set of data of 342 patients by bootstrap validation method, which presented good accuracy for predicting CSS of patients with a bootstrap corrected C-index of 0.742 (95\% CI: 0.711-0.773). The

calibration curves for 1-, 2- and 3-year CSS estimates showed good correlation between the CSS estimates from the nomogram and those derived from Kaplan-Meier estimates (Figure 4).

Table 2. Multivariable Cox model of CSS after forward selection of variables with $P<0.20$ in univariate Kaplan-Meier analyses.

\begin{tabular}{lccc}
\hline Characteristics & HR & CI & P \\
\hline Age, years & & & \\
\hline$\geq 60$ vs. $<60$ & 1.398 & $1.081-1.809$ & .011 \\
\hline $\begin{array}{l}\text { Tumor grade } \\
\text { Grade III, IV vs. Grade I, II }\end{array}$ & 1.623 & $1.246-2.113$ & .000 \\
\hline CEA & & & \\
\hline+ vs. - & 1.626 & $1.158-2.282$ & .005 \\
\hline AJCC N stage & & & \\
\hline N+ vs. N0 & 1.730 & $1.250-2.394$ & .001 \\
\hline $\begin{array}{l}\text { Primary tumor resection } \\
\text { No vs. Yes }\end{array}$ & 2.022 & $1.531-2.669$ & .000 \\
\hline Chemotherapy & & & \\
\hline No vs. Yes & 3.920 & $2.947-5.215$ & .000 \\
\hline HR: hazard rate; CI: confidence interval & & \\
\hline
\end{tabular}
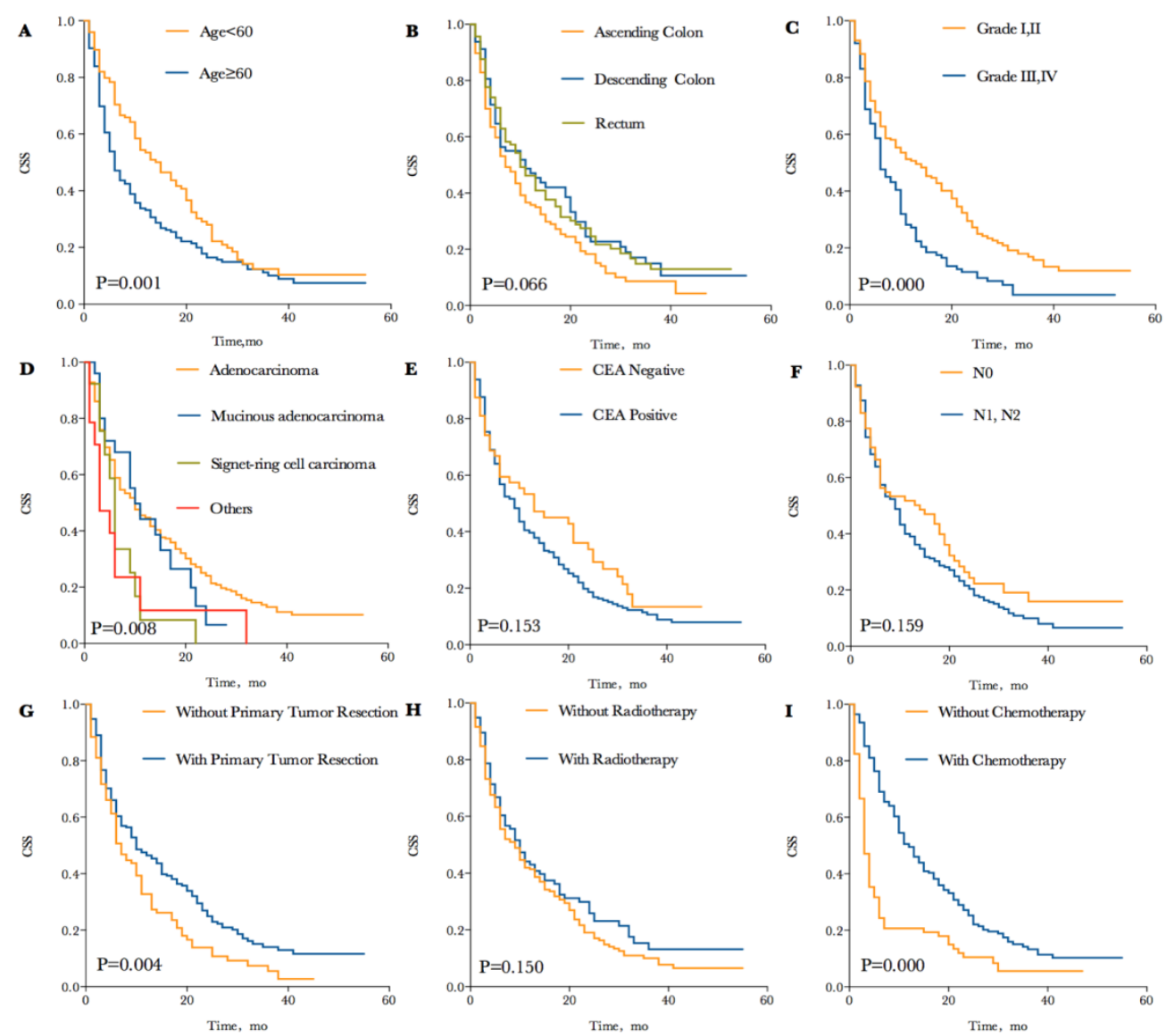

Figure 2 Kaplan-Meier cancer specific survival curves according to different potential variables with $P<0.20$. (A) Kaplan-Meier cancer specific survival curve according to age. (B) Kaplan-Meier cancer specific survival curve according to tumor site. (C) Kaplan-Meier cancer specific survival curve according to tumor grade. (D) Kaplan-Meier cancer specific survival curve according to pathological type. (E) Kaplan-Meier cancer specific survival curve according to CEA level. (F) Kaplan-Meier cancer specific survival curve according to $\mathrm{N}$ stage. (G) Kaplan-Meier cancer specific survival curve according to primary tumor resection. $(\mathrm{H})$ Kaplan-Meier cancer specific survival curve according to radiotherapy. (I) Kaplan-Meier cancer specific survival curve according to chemotherapy. 
Points

Age (years)

AJCC N stage

Grade

CEA

Primary Tumor Resection

Chemotherapy



Total Points

1-year survival

2-year survival



3-year survival
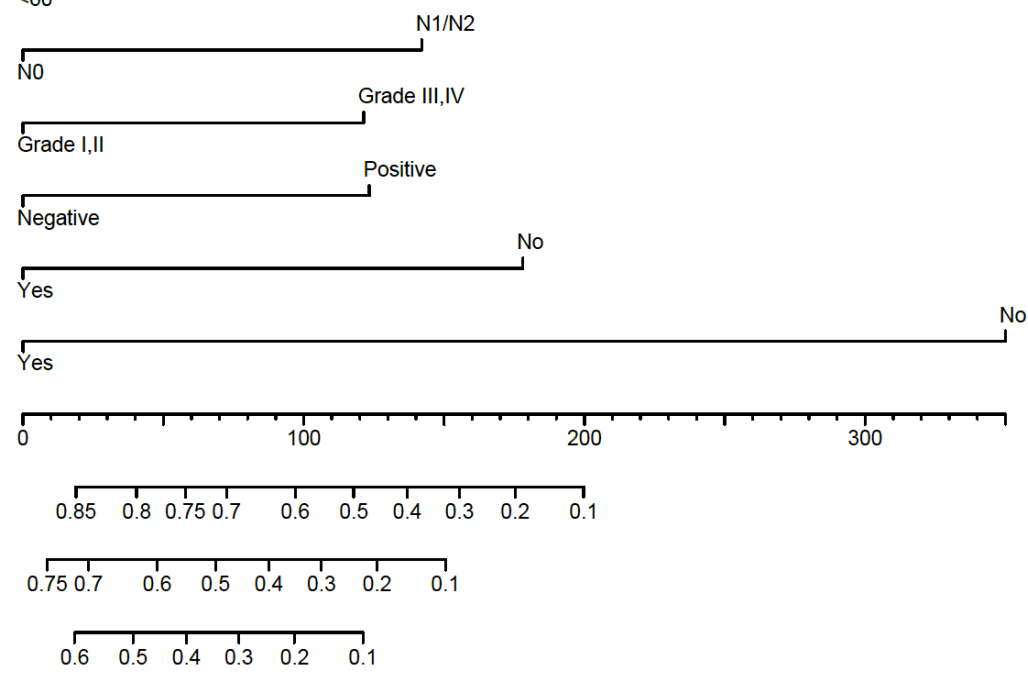

Figure 3 Nomogram for predicting 1-, 2- and 3-year cancer specific survival. Sum the points of each variable (age, AJCC N stage, grade, CEA, primary tumor resection and chemotherapy) and locate this sum on the Total Points axis. Then respectively draw the "1-year survival", " 2 -year survival" and " 3 -year survival" axis to find the predicted cancer specific survival time.

\section{Discussion}

Effective prognostic assessment is crucial for CRC patients diagnosed with synchronous BM. However, there is no complete evaluating system to accurately estimate the prognosis of this group of patients for physicians ${ }^{(6-8)}$. The nomogram, a simple statistical predictive tool, is able to predict the survival and prognosis of $\mathrm{BM}$ more accurately for CRC patients with visualization results, which can further improve the compliance and therapeutic effect of patients. The SEER database provides potent data support for developing the nomogram.

Our study retrospectively analyzed the survival outcomes of total 342 CRC patients with synchronous BM. We finally identified variables including patients' age, tumor grade, CEA levels, AJCC N stage, primary tumor resection and chemotherapy as independent prognostic factors. Then we used these significant factors to develop nomogram. Because the nomogram only included six common clinicopathological variables, it could be used to accurately make precise prediction of survival and guide useful treatment. In addition, the C-index and internal validation also demonstrated this nomogram was a reliable tool for estimate of CSS in CRC patients with synchronous BM.

Here, we could find the prognosis of synchronous $\mathrm{BM}$ in CRC patients was very poor, which is consistent with previous reports. Patients older than 60 years would likely have a worse survival outcomes than younger patients. The CSS in patients with low tumor grade would be longer than in those with high tumor grade. CEA levels positive is associated with worse prognosis and the prognosis of patients with lymphatic metastasis might be worse than those without. Currently, there exists controversy regarding the long-term benefit of primary tumor resection in stage IV and many prior studies suggest there is no benefit to survival with primary tumor resection $(15,16)$, while others suggest a clinical benefit with surgical resection (17-19). In our study, we found the patients could significantly be beneficial from primary tumor resection with remarkable improvement of CSS. That might be because removal of primary tumor could prevent future tumor related complications including bleeding, obstruction and perforation, thereby avoiding the decreased quality and survival of patients' life. Notably, the chemotherapy also was a striking prognostic factor for BM that had been demonstrated in many reports $(12,20,21)$. Points for chemotherapy extended across the full range of point axis, representing much more points than any other variables, which demonstrated the relationship between normalized chemotherapy and better survival. 

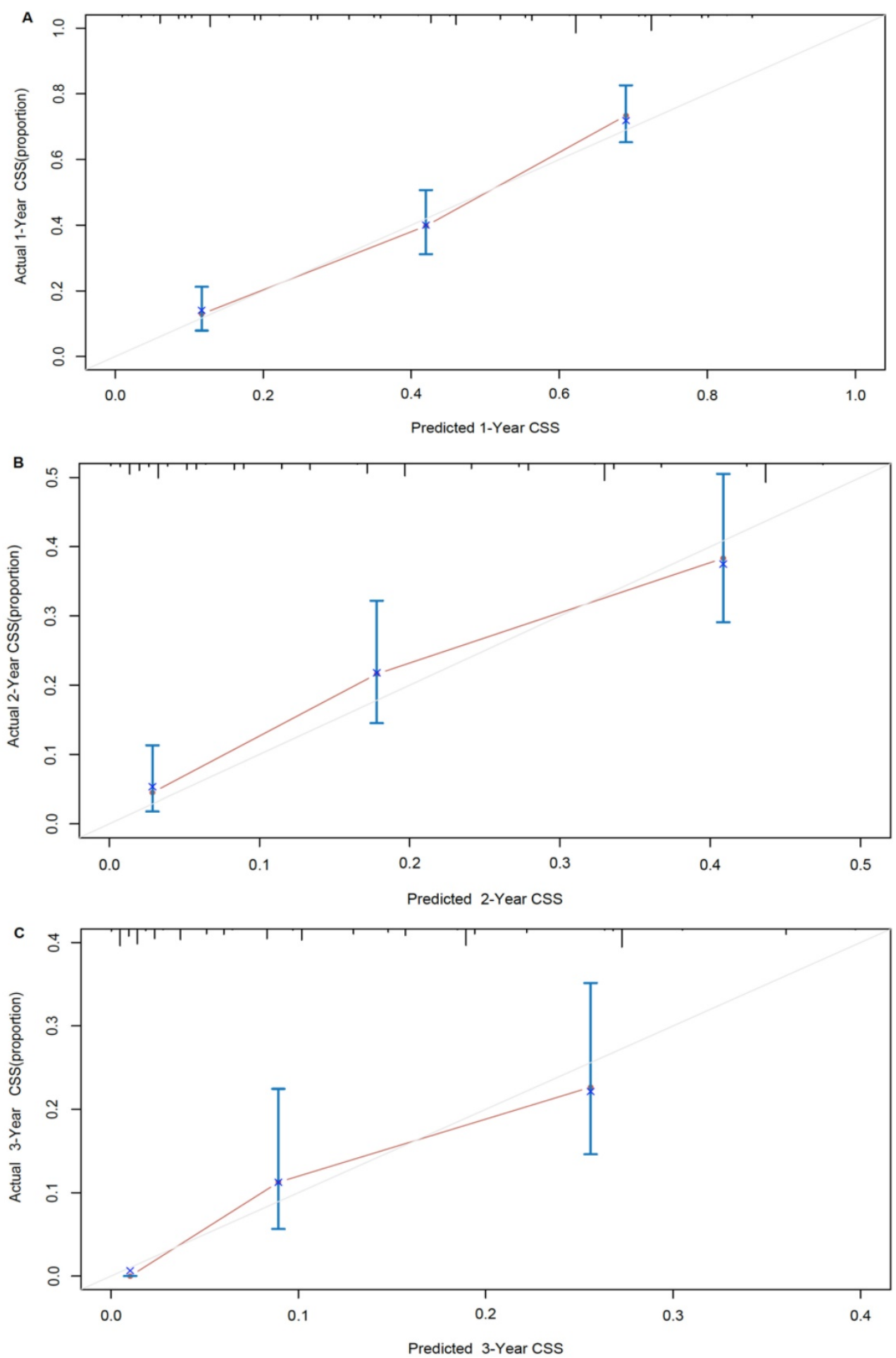

Figure 4 Nomogram model calibration curves of actual cancer specific survival with $95 \%$ confidence interval by decile (y-axis), over predicted cancer specific survival (x-axis) by nomogram: (A) 1-year nomogram calibration curve. (B) 2-year nomogram calibration curve. (C) 3-year nomogram calibration curve.

For example, a 70-year-old (25 points) synchronous BM patients from CRC with lymphatic metastasis (40 points), grade II (0 points), CEA positive (35 points) who undertake primary cancer surgery ( 0 points) and chemotherapy ( 0 points) has a total of 100 points, resulting the estimated 1-, 2-, 3 -year CSS of $58.0 \%, 32.0 \%$ and $18.3 \%$, compared to the total of 250 points with estimated 1-year CSS of less than $10 \%$ if the same patients don't receive neither surgery or chemotherapy. The 1-, 2- and 3 -year CSS of patients with chemotherapy were both remarkably improved combined with surgical resection of primary tumor, which could further guide the treatment of CRC patients with 
synchronous BM.

A Turkish research ${ }^{(22)}$ in 2015 demonstrated that palliative radiotherapy $(p=0.001$, HR 0.51$)$ was an independent prognostic factors for survival of patients with BM,. Other researches have also proved radiotherapy could improve the patients' prognosis $(12,23)$. However, our nomogram showed an interesting result: The radiotherapy failed to be one of independent prognostic factors. So we held the view that radiotherapy had better efficacy in relieving bone pain rather than in treating BM, and there was no significant difference in CSS between patients with and without radiotherapy.

Our finding showed that chemotherapy had a striking impact on the prognosis and could significantly improve CSS of patients. However, it was unclear about the relevant detailed information for chemotherapy regimen and cycle in the SEER database. In addition, because the SEER database only represents the population in the US, which lacks data on Asian population or other races, leading to the limitation in its widespread use.

\section{Conclusions}

Although the patients with synchronous BM only accounted for a small proportion in CRC patients, the incidence has increased in recent years with poor prognosis. Accurate assessment of prognosis and survival at the diagnose of CRC with synchronous BM would be beneficial for patients. Here, the nomogram based on readily available clinicopathologic factors presented high accuracy to precisely estimate the individualized CSS, which could further provide better decision making to both physicians and patients.

\section{Acknowledgements}

The authors acknowledge the great efforts of the Surveillance, Epidemiology, and End Results (SEER) Program in the creation of the SEER database.

\section{Competing Interests}

The authors have declared that no competing interest exists.

\section{References}

1. Freddie B, Jacques F, Isabelle S, et al. Global Cancer Statistics 2018: GLOBOCAN Estimates of Incidence and Mortality Worldwide for 36 Cancers in 185 Countries. CA: A Cancer Journal for Clinicians. 2015.

2. O'Connell JB, Maggard MA, Ko CY. Colon Cancer Survival Rates With the New American Joint Committee on Cancer Sixth Edition Staging. Journal of the National Cancer Institute. 2004;96:1420.

3. Sundermeyer ML, Meropol NJ, Rogatko A, et al. Changing Patterns of Bone and Brain Metastases in Patients with Colorectal Cancer. Clin Colorectal Cancer. 2006;5:108-13.

4. Khattak MA, Martin HL, Beeke C, et al Survival Differences in Patients With Metastatic Colorectal Cancer and With Single Site Metastatic Disease at Initial Presentation: Results From South Australian Clinical Registry for Advanced Colorectal Cancer. Clinical Colorectal Cancer. 2012;11:247-54.

5. Coleman RE. Skeletal complications of malignancy. Cancer. 2010;80:1588-94.
6. Kawamura H, Yamaguchi T, Yano Y, et al. Characteristics and Prognostic Factors of Bone Metastasis in Patients With Colorectal Cancer. Diseases of the Colon \& Rectum. 2018;61:673-8.

7. Nozue M, Oshiro $\mathrm{Y}$, Kurata M, et al. Treatment and prognosis in colorectal cancer patients with bone metastasis. Oncology Reports. 2002;9:109-12.

8. Baek SJ, Hur H, Min BS, et al. The Characteristics of Bone Metastasis in Patients with Colorectal Cancer: A Long-Term Report from a Single Institution. World Journal of Surgery. 2015;40:1-5.

9. Bostel T, Förster R, Schlampp I, et al. Spinal bone metastases in colorectal cancer: a retrospective analysis of stability, prognostic factors and survival after palliative radiotherapy. Radiation Oncology. 2017;12:115.

10. Baek SJ, Hur H, Min BS, et al. The Characteristics of Bone Metastasis in Patients with Colorectal Cancer: A Long-Term Report from a Single Institution. World Journal of Surgery. 2016;40:1-5.

11. Wenhua L, Li Z, Gening J, et al. Development and validation of a nomogram for predicting survival in patients with resected non-small-cell lung cancer. Journal of Clinical Oncology. 2015;33:861-9.

12. Vincenzo V, Stiphout RGPM, Van, Guido L, et al. Nomograms for predicting local recurrence, distant metastases, and overall survival for patients with locally advanced rectal cancer on the basis of European randomized clinical trials. Journal of Clinical Oncology Official Journal of the American Society of Clinical Oncology. 2011;29:3163.

13. Zaak D, Burger $M$, Otto $W$, et al. Predicting individual outcomes after radical cystectomy: an external validation of current nomograms. Bju International. 2010;106:342-8.

14. Kang SJ, Cho Y-R, Park G-M, et al. Predictors for Functionally Significant In-Stent Restenosis. Jacc Cardiovascular Imaging. 2013;6:1183-90.

15. Matsumoto T, Hasegawa S, Matsumoto S, et al. Overcoming the Challenges of Primary Tumor Management in Patients With Metastatic Colorectal Cancer Unresectable for Cure and an Asymptomatic Primary Tumor. Diseases of the Colon \& Rectum. 2014;57:679-86.

16. Massarweh NN, Li LT, Sansgiry S, et al. Primary Tumor Resection and Multimodality Treatment for Patients with Metastatic Colon Cancer. Annals of Surgical Oncology. 2016;23:1815-23.

17. Park J-H, Kim T-Y, Lee K-H, et al. The beneficial effect of palliative resection in metastatic colorectal cancer. British Journal of Cancer. 2013;108:1425-31.

18. Gulack BC, Nussbaum DP, Keenan JE, et al. Surgical Resection of the Primary Tumor in Stage IV Colorectal Cancer Without Metastasectomy Is Associated With Improved Overall Survival Compared With Chemotherapy/Radiation Therapy Alone. Diseases of the Colon \& Rectum. 2016;59: 299.

19. Maroney S, Paz CCd, Reeves ME, et al. Benefit of Surgical Resection of the Primary Tumor in Patients Undergoing Chemotherapy for Stage IV Colorectal Cancer with Unresected Metastasis. J Gastrointest Surg. 2018;22:460-6.

20. Van CE, Cervantes A, Adam R, et al. ESMO consensus guidelines for the management of patients with metastatic colorectal cancer. Annals of Oncology. 2016;27:1386-422.

21. Hirotoshi K, Kenjiro K, Kenichi S. Impact of adjuvant chemotherapy in patients with curatively resected stage IV colorectal cancer. Medicine. 2015;94:e696.

22. Ulas A, Bilici A, Durnali A, et al. Risk factors for skeletal-related events (SREs) and factors affecting SRE-free survival for nonsmall cell lung cancer patients with bone metastases. Tumor Biology. 2016:37:1131-40.

23. Edward C, Kristin H, Grace F, et al. Palliative radiotherapy trials for bone metastases: a systematic review. Journal of Clinical Oncology Official Journal of the American Society of Clinical Oncology. 2007;25:1423. 